\title{
e-Migrinter
}

$7 \mid 2011$

Réflexions croisées sur les migrations en Afrique de l'Ouest

\section{Histoire des migrations internationales et des luttes contre les discriminations}

Compte-rendu du Cycle de conférence de l'Espace Mendès France, Poitiers 2010-2011

\section{Mickaël Quintard}

\section{(2) OpenEdition}

Journals

Édition électronique

URL : https://journals.openedition.org/e-migrinter/908

DOI : 10.4000/e-migrinter.908

ISSN : 1961-9685

Éditeur

UMR 7301 - Migrinter

Édition imprimée

Date de publication : 1 septembre 2011

Pagination : 86-88

ISSN : 1961-9685

Référence électronique

Mickaël Quintard, « Histoire des migrations internationales et des luttes contre les discriminations », eMigrinter [En ligne], 7| 2011, mis en ligne le 13 septembre 2017, consulté le 20 mai 2021. URL : http:// journals.openedition.org/e-migrinter/908; DOI : https://doi.org/10.4000/e-migrinter.908 


\section{Cycle de conférence sur l'histoire des migrations internationales et des luttes contre les discriminations, Espace Mendès France, Poitiers 2010-2011}

Compte-rendu par Mickaël Quintard

$\mathbf{L}$ e cycle de conférence 20102011 organisé par 1'Espace Mendès-France de Poitiers s'est appuyé sur l'aide du réseau des chercheurs et universitaires du laboratoire Migrinter avec le soutien de l'Acsé. Un ensemble de séances ont été organisées autour de sept intervenants pour la plupart universitaires. Des thématiques variées ont été abordées, de l'asile (Luc Legoux et Smaïn Laacher), aux Gens du Voyage (Céline Bergeon et Christian Quella) en passant par des sujets plus larges avec la conférence inaugurale de Gildas Simon ou des problématiques plus précises inspirées d'étude de cas : la question du genre à travers le cas de migrantes marocaines en France (Nasima Moujoud) ou des trajectoires de mobilité sociale et professionnelle des migrants tunisiens (Anne-Sophie Bruno).

Nous avons choisi pour $e$ migninter de restituer les interventions de Gildas Simon et de Luc Legoux.

\author{
Conférence de Gildas Simon : "Histoire \\ coloniale et mondialisation des \\ migrations internationales »
}

La réflexion de Gildas Simon s'articule autour des trois notions que sont la colonisation, la mondialisation et la migration internationale. Après avoir rappelé leurs définitions, la réflexion s'oriente autour du rôle des colonisations et décolonisations dans la genèse des migrations internationales, et plus précisément des articulations anciennes entre les métropoles des anciens pays colonisateurs et les anciens territoires colonisés; puis autour de celui de la mondialisation dans les nouvelles formes de migrations internationales.

C'est avec le cas algérien que Gildas Simon tente de montrer le rôle de la colonisation au sein de la genèse des migrations internationales. Il montre notamment que les premières migrations kabyles ne correspondent pas à des migrations spontanées puisqu'elles rentrent dans le cadre de "recrutements» organisés par des entreprises marseillaises. Il revient également sur la nécessité d'inscrire la réflexion dans le temps long, comme le montre l'écart important entre le début de la colonisation (1830) et le début des migrations de certains Algériens en direction de la France. Si les migrants algériens constituaient la base de l'immigration ouvrière en France, la première guerre mondiale fut un véritable tournant dans le développement des migrations internationales qui diffusa l'« onde migratoire » et l'habitude de franchir la Méditerranée était maintenant établie dans les comportements migratoires. 
Gildas Simon aborde ensuite le temps de la décolonisation (1945-1962) et des «Trente Glorieuses ", ainsi que le développement et la consolidation des « couples migratoires ». Fonctionnant sur un modèle colonial, ces couples évoluèrent avec la décolonisation et les évolutions furent différentes selon les pays concernés.

À partir des années 1970, commence le temps de la mondialisation et de l'élargissement du « champ migratoire ». Les couples migratoires n'étaient alors plus exclusifs, le modèle colonial évoluait pour devenir spatialement et culturellement plus diversifié. Des phénomènes de «report» apparurent, certains migrants réorientant alors leurs parcours selon les politiques des pays d'accueil. À ce titre, l'Espagne et l'Italie devinrent des pôles forts attractifs. Le lien colonial perdit de l'importance au profit de logiques économiques nationales, mais également au profit de la logique de mondialisation. Si la logique coloniale a pu rendre compte de la genèse des migrations internationales et de leur intensification, elle s'est peu à peu effacée pour laisser place à d'autres logiques. $\mathrm{Si}$ les migrations internationales ont des substrats historiques et culturels liés à la colonisation; c'est à d'autres dynamiques fondamentales qu'obéissent aujourd'hui les comportements migratoires. Le facteur économique est devenu déterminant, comme en témoigne la proportionnalité entre nombre de migrants et niveau de richesse des pays. Le «mythe de l'eldorado » est aujourd'hui plus vivant que jamais, il est de nos jours notamment entretenu par les moyens de communication tels que la télévision et internet.

En conclusion, Gildas Simon rappelle que la migration n'est pas seulement une question interrogeant les décideurs, c'est également une réponse. En effet, dans un monde divisé par de fortes inégalités économiques et sociales, les migrations constituent une réponse pour accéder à la dignité que certains ne peuvent atteindre dans leurs pays.
Conférence de Luc Legoux : " $L a$ soumission par les mots : étude des catégories administratives des migrants et des réfugiés »

Dans le cadre de sa conférence « La soumission par les mots : étude des catégories administratives des migrants et des réfuiiés 》 à l'espace Mendès France à Poitiers, le 12 novembre 2010, Luc Legoux commence par rappeler le cadre général du vocabulaire. Du point de vue de l'accueil des États, l'asile est une forme d'ingérence dans un conflit, ce qui l'inclut dans la problématique des relations internationales. À l'origine lié aux sanctuaires de l'Antiquité (sa pratique confortant alors le pouvoir en place), l'asile est présenté comme un élément de la défense des droits de l'homme de la Révolution française jusqu'à 1989. Il est aujourd'hui davantage lié à des sentiments humanitaires, liaison correspondant alors à un habillage idéologique ayant tendance à faire oublier un certain égoïsme des États. Il est ici mentionné l'importance du vocabulaire dans ces processus d'instrumentalisation.

L'exposé s'organise autour de cinq expressions, et ce afin de montrer l'évolution $\mathrm{du}$ vocabulaire et son importance : l'immigration politique, les réfugiés politiques, les demandeurs d'asile, la protection subsidiaire et les réfugiés climatiques.

L'immigration politique est un terme largement employé au début du régime d'asile (Convention de Genève, 1951), lequel sous-entend que l'immigration a un caractère politique. A l'époque, l'association de ces deux termes est favorable à l'accueil d'autant que les besoins en main-d'œuvre sont élevés. C'est en 1978 que l'appellation d' « immigration politique » disparait dans le langage tant des institutions que des associations.

C'est ensuite la phase de l'emploi du terme de «réfugié politique». Celui-ci, 
même s'il n'a aucune valeur juridique, véhicule une image positive dans l'opinion et est investi d'une certaine respectabilité. Mais l'opposition entre réfugié politique et réfugié économique grandit, et la terminologie de réfugié politique disparaît en 1986. On assiste alors à un certain abandon de la volonté politique au profit d'une compassion humanitaire.

L'expression de «demandeur d'asile » vient remplacer celle de "réfugié en attente de statut » en 1981, année où l'existence de fraudes est découverte (demandes multiples). On ne présuppose alors plus que la personne est réfugiée, mais plutôt qu'elle en capacité d'être déboutée. Avec l'accroissement des demandes rejetées, l'usage du terme se généralise, tout comme la présomption de demande abusive.

La protection subsidiaire est quant à elle le fruit de l'harmonisation des politiques migratoires de l'Union Européenne. Il s'agit de protéger les individus menacés de mort, tortures et menaces graves en raison de violences liées à des conflits armés. Si l'on suit la définition de manière stricte, cette protection ne devrait alors bénéficier qu'à un nombre de personnes relativement restreint, les autres se retrouvant inclus dans le statut de la Convention de Genève. Pourtant, cette protection concerne un nombre d'individus toujours plus important. Cette mesure qui théoriquement devait être complémentaire de la Convention de 1951, devient peu à peu une mesure de substitution et entraîne une précarisation du statut puisque par exemple la protection est désormais temporaire.
Enfin, l'émergence de l'expression de «réfugiés climatiques» renvoie à trois enjeux : celui de la gestion des migrations au niveau mondial - lié au nombre important de personnes concernées -, de la protection des réfugiés politiques et du développement des organisations appelées à gérer ces mouvements migratoires. Le risque pour le sujet des réfugiés politiques étant que le développement d'accords de coopération permettrait de refuser l'asile, notamment par le biais d'une protection apportée sur place. L'émergence de cette expression est donc une aubaine pour les pays du Nord dans une perspective de fermeture des frontières. Le risque majeur étant celui d'un affaiblissement du système juridique international de protection.

En conclusion, Luc Legoux rappelle que les mots orientent plus qu'ils ne décrivent et qu'il est aujourd'hui nécessaire de résister au langage dominant.

Mickaël Quintard

Doctoranten Géographie Migrinter UMR 6588

CNRS - Université de Poitiers mickael.quintard@,univ-poitiers.fr 